\title{
A review on antifungal agents against Candida albicans and Aspergillus niger
}

\author{
Vishnuvardhini R and Priya R Iyer * \\ Post-Graduation \& Research Department of Biotechnology, Women's Christian College, An Autonomous Institution \\ Affiliated to the University of Madras, Chennai-600006, Tamil Nadu, India.
}

International Journal of Biological and Pharmaceutical Sciences Archive, 2021, 02(01), 042-054

Publication history: Received on 18 June 2021; revised on 22 July 2021; accepted on 24 July 2021

Article DOI: https://doi.org/10.53771/ijbpsa.2021.2.1.0061

\begin{abstract}
Candidiasis is the fungal infection caused by Candida albicans. It causes different type of candidiasis infections in the blood, heart, eyes, brain, bones also other parts of our body. Different types of candidiasis are vaginal candidiasis, pulmonary candidiasis, oral candidiasis. It may be acute or chronic. It can be cured by using natural compounds or synthetic compounds or semisynthetic compounds. Apergillosis is the fungal infections in human caused by Aspergillus niger. Aspergillosis infections are classified into allergic pulmonary sinusitis, cutaneous aspergillosis, invasive aspergillosis, chronic necrotising pulmonary aspergillosis can be cured by using synthetic compounds like intravenous injections, oral medicines of capsules, tropical medicines like creams and some other natural products from plants. Natural products like saponins, flavonoids, alkaloids, xanthones, lectins and polypeptides, quinones, terpenoids, coumarins, essential oils and other compounds are the secondary metabolites extracted from the plants. Those plants are effective against fungal infections. Synthetic drugs like Azole group of drugs composed of imidazoles which includes miconazole, clotrimazole, ketoconazole, and triazoles include itraconazole, Posaconazole, isavuconazole are used to inhibit the ergosterol synthesis of cell membrane of fungi. Echinocandins are antifungal agent and it is used to disrupt the cell wall of fungi and inhibiting the synthesis of beta- 1,3 glucan fungal cell wall. Echinocandins are classified into caspofungin, anidulafungin, micafungin which having antifungal activity and amphotericin B used to treat both candidiasis and aspergillosis. Combination of drugs like synthetic drugs and natural compounds used to treat Candidiasis and Aspergillosis. Comparison and effectiveness of drugs depends upon the infections they are getting affected. Amphotericin B and azole group of drugs are commonly more effective than other group of drugs, but ketoconazole is widely used. Adverse effects of antifungal agents include, skin rashes, irritation, itching, nausea and vomiting, fever, abdominal pain, muscle and joint pain, etc.
\end{abstract}

Keywords: Candidiasis; Aspergillosis; phenols; alkaloids; amphotericin B; fluconazole

\section{Introduction}

Candidiasis is the fungal infection caused by yeast called candida. Different types of candida species are there, in that Candida albicans is the one of the fungal species that can cause infections in human body.(Martins et al., 2014),(Papon et al., 2013). It lives on the skin, inside the body, throat, mouth, intestine, vagina without causing any problems. If Candida albicans grows out of the control and deep inside the body, causing infections. It causes in the blood stream, brain, gut, human reproductive system of the bod. Systemic candidiasis includes a spectrum of fungal infections caused by Candida albicans. It is the infection caused in the blood, heart, eyes, brain, bones also other parts of our body. Systemic candidiasis may also affect central nervous system, brain, spinal cord, muscles, joints, spleen, bones, kidneys, abdomen and the symptoms depend upon the organ infected. For example, if the candida infects eye, symptoms may be blurred vision with photophobia(Martins et al., 2014).

\footnotetext{
${ }^{*}$ Corresponding author: Priya R Iyer

Post-Graduation \& Research Department of Biotechnology, Women`s Christian College, An Autonomous Institution Affiliated to the University of Madras, Chennai-600006, Tamil Nadu, India.
} 


\subsection{Oral candidiasis}

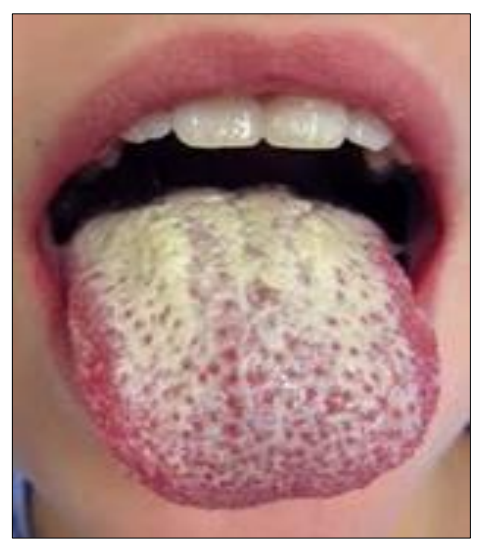

Figure 1 Oral candidiasis

It is an infection caused by accumulation of Candida albicans in mouth. It is otherwise called as oropharyngeal candidiasis, candidal stomatitis. It grows on the mucous membrane of mouth.(Vila et al., 2020).

\subsection{Traditional classification of oral candidiasis}

It includes acute candidiasis and chronic candidiasis.

\subsection{Acute candidiasis}

Acute candidiasis is further classified into two types: pseudomembranous candidiasis and atrophic candidiasis.

\subsubsection{Pseudomembranous candidiasis}

It is commonly called as thrush. It is characterized by the patches of pseudomembranous white spot like and sometimes it causes bleeding. It can affect any part of mouth, but it usually appears white patches or white coating of the tongue, buccal mucosa. In acute condition, it affects infants, people who having very low immunity and undergone with immunosuppressant medications.(Hellstein \& Marek, 2019).

\subsubsection{Atrophic candidiasis}

Atrophic candidiasis is otherwise known as erythematous which forms red blisters or lesions on the mouth. Erythematous is commonly appears on the buccal mucosae or palate. It is so painful. It occurs on the dorsum of tongue for the person who is taking corticosteroids continuously.(Hani et al., 2015).

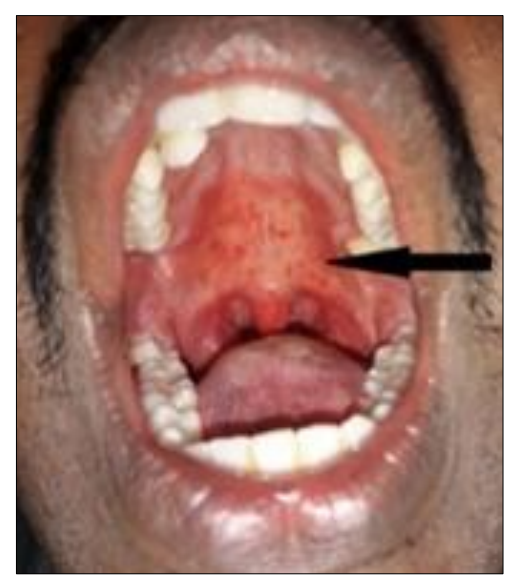


Figure 2 Atropic Candidiasis (Bakshi, 2018)

\subsection{Chronic candidiasis}

\subsubsection{Hyperplastic}

It is termed as "plaque like candidiasis" or "nodular candidiasis". The appearance of the hyperplastic candidiasis is persistent white plaque.(Mohamed et al., 2019)(Rawashdeh et al., 2011).

\subsubsection{Angular cheilitis}

It caused by Candida albicans at the angle or corner of the mouth. It includes soreness, redness, swelling on the corners of the mouth. Candida albicans associated with other species causing angular cheilitis and it is called as associative lesions.(Lelarge \& Mariot, 1992).

\subsubsection{Mediated rhomboid glossitis}

It is an elliptical lesion occurs in the centre of dorsal tongue, circumvallate papillae which is on the upper surface of tongue with rough texture. It is so painful.(Vila et al., 2020).s

\subsubsection{Linear gingival erythema}

The people who affected by AIDS/HIV. It causes inflammation in gum. Necrotizing ulcerative gingivitis are mostly lesions in patients with HIV infection.

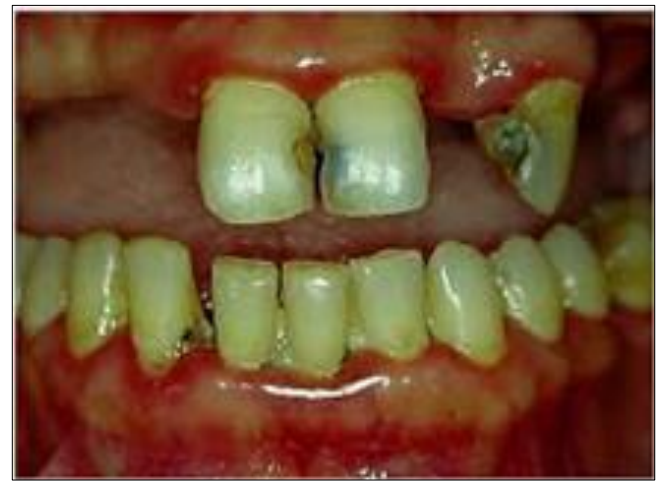

Figure 3 Linear gingival erythema in HIV patient (Panagakos \& Migliorati, 2014)

\subsection{Pulmonary candidiasis}

It is the fungal infection caused by Candida albicans which occurs in lungs. The persons who are affected by cancer, autopsy-proven pneumonia, tuberculosis is having more chances to get pulmonary candidiasis. Candida albicans forms colonies in the mucosal surface of respiratory tract, sputum with blood.(Kontoyiannis et al., 2002).

\subsection{Vaginal candidiasis}

Vaginal candidiasis is the fungal infection caused by Candida albicans. It can multiply and cause infection in the vagina. It is otherwise called as vulvovaginal candidiasis or candida vaginitis. Symptoms are sometimes, during pregnancy blisters of Candida albicans are found in vagina. It can survive even in acidic conditions. Some women can develop infections like redness, swelling. Vaginal itching, paining, discomfort when urinating.(Leli et al., 2013),(Kauffman, 2014).

\subsection{Aspergillosis}

Aspergillosis is the infection caused by fungi called Aspergillus niger. It causes infections in lungs and it also causes several infections like chronic necrotizing pulmonary aspergillosis. Chronic necrotizing pulmonary aspergillosis is otherwise called as semi-invasive pulmonary aspergillosis. The people who are having low immunity are getting this infection.(Kobashi et al., 2006). 


\subsection{Allergic bronchopulmonary aspergillosis}

It can be caused by inflammation of Aspergillus in lungs and causes infection, allergies and some symptoms like cough, wheezing.(Greenberger et al., 2014),(Agarwal et al., 2016).

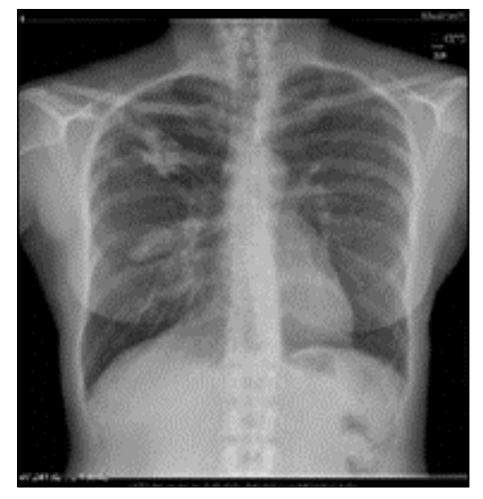

Figure 4 Allergic bronchopulmonary Aspergillosis (Greenberger et al., 2014)

\subsection{Allergic aspergillosis sinusitis}

Allergic aspergillosis sinusitis can be caused by inflammation of Aspergillus in sinuses mucosal membrane contained in eosinophil. The symptoms are headache, nasal blockage.(Chakrabarti \& Kaur, 2016),(Katzenstein et al., 1983).

\subsection{Invasive aspergillosis}

The people who undergone with organ transplant, stem cell transplant especially hematopoietic stem transplant and also having very low immunity are getting invasive aspergillosis.(McCarty \& Pappas, 2016), (Zmeili \& Soubani, 2007).

\section{Natural compounds}

Natural products which are used to treat against fungal infections like Candidiasis and Aspergillosis. Natural products like saponins, flavonoids, alkaloids, xanthones, lectins and polypeptides, quinones, terpenoids, coumarins, essential oils and other compounds are the secondary metabolites extracted from the plants. Those plants are effective against fungal infections.(Arif et al., 2009).

\subsection{Phenols}

Phenols is the natural compound extracted from the plants, it having hydroxyl group so it undergoes hydroxylation and it causes toxicity to microorganisms. Tannins and salicylic acid are polyphenolic compounds which are extracted from Gaultheria procumbens (eastern boxberry, checkerberry, teaberry plant), Rhamnus purshiana (cascara plant belongs to Rhamnaceous family), Anacardium pulsatilla (flowering plant, usually used as homeopathic remedies) showed antifungal activity. (Arif et al., 2009).(Haidara et al., 2016).

\subsection{Flavonoids}

Group of flavones are the structure of phenolic compounds that containing "1" carbonyl group and "3" hydroxyl group to yield flavonoids. Fig.5. Flavonoid from Alpinia officinarum (root of the plant belongs to ginger family) are effective against antifungal activity.(Antifungal Flavonoid from Alpinia Officinarum Hance - PubMed, n.d.). Isoflavan, 2-hydroxy maackiain extracted from the Hildegardiabarteri (root of the plant belongs to Malvaceae family).(Meragelman et al., 2005). 


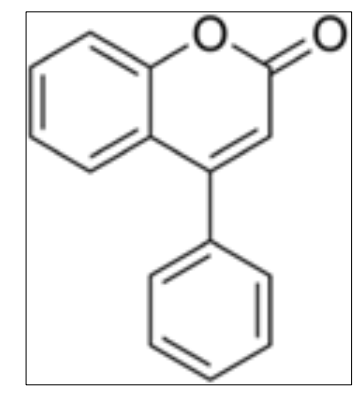

Figure 5 Flavonoid (McNaught et al., 2008).

\subsection{Coumarins}

Coumarins are composed by phenolic compounds with fusion of benzene and alpha pyrone rings. (Arif et al., 2009). Coumarin from the licorice of Glycyrrhizae radix (flowering plant of the bean belongs to Fabaceae) having antifungal activity especially inhibited the cell wall synthesis of glucan in Candida albicans.(Rhew\& Han, 2016). Angelicin are phytochemical compound which are naturally synthesized furanocoumarin showed antifungal activity against Aspergillus niger and Candida albicans. (Sardari et al., 1999).

\subsection{Quinones}

Quinones which are made up of aromatic rings with substitutions of 2 ketone groups. (Arif et al., 2009). Fig6. Alphamangostin and lawsone methyl ether (2-methoxy-1,4-napthoquinone)are phytochemicals which are derived from the plants are used to treat oral candidiasis.(Nittayananta et al., 2018). Emodin (6-methyl-1,3,8-trihydroxyantraquinone) which inhibit the cell wall synthesis of $(1,3)$ - beta-D- glucan of fungi which are mainly used to inhibit the growth of Candida albicans.(Janeczko, 2018).

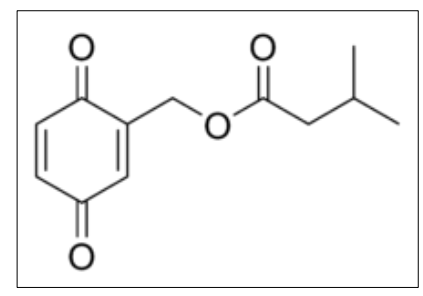

Figure 6 Structure of quinone (Patai \& Rappoport, 2010)

\subsection{Saponins}

Saponins are secondary metabolites which are extracted from the plant. Saponins are inactive precursors by adding enzymes it is converted into active compound corresponding to pathogens attack. Saponins are glycosylated compound and it can be classified into 3 major groups; namely a triterpenoid, a steroid, a steroidal glycoalkaloid. These compounds are helps to inhibit the growth of fungi especially Candida albicans. Steroidal saponins are from the root of Smilaxmedica used to treat fungal infections. (Arif et al., 2009)(Belhouchet et al., 2008).

\subsection{Xanthones}

Xanthones composed of benzene rings attached with carbonyl group and oxygen are bioactive compounds which are extracted from the plant used to treat fungal infection. 1,3,6-Trihydroxy-2,5-dimethoxyxanthone is extracted from the aerial part of Monninaobtusifolia (polygalaceae family) against fungal infections (Arif et al., 2009)(Hay et al., 2003). Seven xanthanolides are isolated from Xanthium macrocarpum having antifungal property.(Lavault et al., 2005).

\subsection{Alkaloids}

Alkaloids are made up of heterocyclic nitrogen compounds. Morphine is a kind of alkaloid isolated from the opium poppy Papaver somniferum.(Dharmaratne et al., 2005).Fig.7. 2-(3,4-dimethyl2,5-dihydro-1H-pyrrol-2-yl)-1methylethyl pentanoate is an alkaloid which are extracted from the plant Datura metel and having In vitro as well as in vivo activities against Aspergillus niger and Candida albicans. An alkaloid is extracted from the plant Anibapanurensis having antifungal activity. 


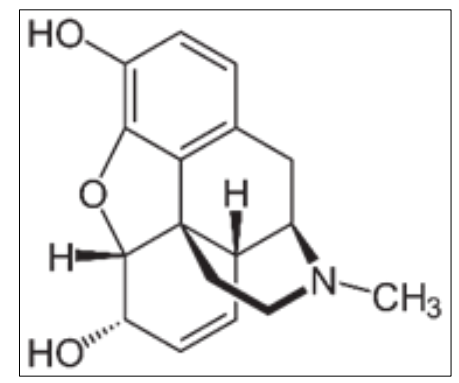

Figure 7 Structure of morphine (Novak et al., 2005)

\subsection{Lectins and polypeptides}

Peptides which are used to inhibit the growth of microorganism. A lectin and a polypeptide which are extracted from the roots of Astragalus mongholicus active against fungi. (Lam \& Ng, 2011). An antifungal peptide is extracted from the mushroom Agrocybecylindracea having antifungal activity. A peptide called cicerarin which are isolated from the seeds of the green chickpea Cicer arietinum are used to inhibit the growth of fungi.(Chu et al., 2003). An antifungal peptide called angularin which are isolated from the adzuki bean are active against fungi. (Lam, Sze Kwan Ng, Tzi Bun, 2003).

\subsection{Terpenoids and essential oils}

Terpenoids and essential oils are phytochemicals which are extracted from source of plants.The essential oil and the methanolic leaf extracted from Teucrium sauvagei inhibited the growth of dermatophytes (fungal infection on skin).(Hayta, Yazgin and Bagci, 2017). Oleanane triterpenoid, triterpenetetrol isolated by using chloroform in Leontodon filiiplant and used to inhibit the growth of fungi. (Arif et al., 2009).Volatile oils are extracted from lemon grass of Cymbopogon citratus (belongs to Poaceae family) used to treat against aspergillosis by Aspergillus niger. (Boukhatem et al., 2014).

\subsection{Bioactive molecules from marine species}

Pentacyclic compound which are extracted from Okinawan marine sponge Xestospongiasapra which are active against Candida albicans. Anthracycline related compounds which are extracted from marine plants, algae, sponges, and soft corals are used to treat fungal infections. (Spampinato \& Leonardi, 2013).

\section{Synthetic compounds}

Synthetic compounds like azole group of drugs and echinocandin group of drugs which are used to cure fungal infections in skin, mouth, gastrointestinal tract and vagina etc.

\subsection{Azole group of drugs}

Azole group of drugs composed of imidazoles which includes miconazole, clotrimazole, ketoconazole, and triazoles include itraconazole, Posaconazole, isavuconazole that are named by nitrogen atoms present in the azole ring. Those azole group of drugs are used to inhibit the ergosterol synthesis of cell membrane of fungi. Azole group of drugs are disrupting the cell wall by accumulation of sterol precursors and as a result ergosterol can be inhibited. Azole group of drugs are mainly used to treat systemic candidiasis and vulvovaginal candidiasis and chronic pulmonary Aspergillosis.(Saag \& Dismukes, 1988), (Bodey, 1992).

Itraconazole are used to treat fungal infections in nail. Itraconazole along with fluconazole, terbinafine, ketoconazole and griseofulvin, used for the treatment of fungal infections in skin (Korting \& Schöllmann, 2009). Itraconazole and fluconazole are the first generation of triazole and voriconazole, Posaconazole, isavuconazole, albaconazole, ravuconazole, efinaconazole are the second generation of triazole are antifungal agents which are used to treat against Candidiasis and Aspergillosis. (Peyton et al., 2015). Itraconazole is used as capsule which is used to treat fungal infections in nails, skin, kidney, lungs, liver, gastrointestinal tract etc. (Willems et al., 2001). Fluconazole is the one of the azole group of drugs which are used to inhibit the fungal cytochrome P-450 3-A dependent enzyme 14-alpha demethylase, interrupting the synthesis of fungal cell membrane called ergosterol, further the cell membrane of ergosterol started to depleted. (Bersani et al., 2019). 
Voriconazole is the first line therapy used to treat invasive Aspergillosis and Chronic necrotizing pulmonary Aspergillosis. (Sambatakou et al., 2006). Voriconazole is the synthetic drug which disrupting the cell membrane by cytochrome P450 (CYP) dependent enzyme 14-alpha -sterol demethylase. Voriconazole is given as oral or intravenous drug to adults, neonates to trat the fungal infections. (Scott \& Simpson, 2007).

Isavuconazole has antifungal activity against Candida albicans and Aspergillus niger. It is given as both oral and intravenous drug and it distributes evenly in body tissues and eliminated slowly. It reaches the target and cure the infection quickly. Instead of voriconazole, isuvuconazole can be used to cure invasive mould infections. Voriconazole is usually used to treat invasive Aspergillosis. (Pettit \& Carver, 2015).Posaconazole (Sch 56592) is a new triazole antifungal agent that can cure both Candidiasis and Aspergillosis. (Pfaller et al., 1997). Posaconazole is used to treat oropharyngeal Candidiasis and also used to treat the people who are getting fungal infections by hematopoietic stem cell transplant.(Smith et al., 2009) . (Rachwalski et al., 2008). Fluconazole, itraconazole are used to treat as chemoprophylaxis of oral medicines to treat the fungal infections. (Meis \& Verweij, 2001).The original azole groups are ketoconazole, miconazole is used as topical preparations for the treatment of vaginal Candidiasis or cutaneous fungal infections.

\subsection{Echinocandin group of drugs}

Echinocandins are composed of cyclic hexapeptides N-linked to a fatty acyl side chain. It is used to disrupt the cell wall of fungi and inhibiting the synthesis of beta- 1, 3 glucan fungal cell wall. Echinocandins are classified into caspofungin, anidulafungin, micafungin which having antifungal activity and against especially Candida albicans. (Grover, 2010). Candida infections can cause extremely low birth weight and sometimes the infants lead to death, blindness. So, Amphotericin $\mathrm{B}$ is given to treat candida infections in neonates or azoles, polyenes are used to inhibit the synthesis of cell wall of fungi and also inhibit the DNA/RNA of fungi.(Clerihew \& McGuire, 2012)(Bersani et al., 2019). Caspofungin, Anidulafungin, Micafungin are the classes of Echinocandins. Echinocandins are used to inhibit the cell wall synthesis by the enzyme called 1,3- beta glucan synthase. In that class, caspofungin is the antifungal drug that are used to cure invasive Candidiasis and Aspergillosis. Anidulafungin, Micafungin drugs are capable of depleting the fungal cell wall and it can be also used to cure invasive fungal infections. (Clerihew \& McGuire, 2012), (Bersani et al., 2019). Micafungin is a lipopeptide compound belongs to the class of Echinocandins that are used to treat invasive Candidiasis, Candidemia and invasive Aspergillosis. (Enoch et al., 2018),(Denning et al., 2006). Liposomal Amphotericin B is formulated by lipid of polyene. It is active against mould or yeast infection caused by Candida albicans and Aspergillus niger. Liposomal Amphotericin B is more effective than Amphotericin B deoxycholate (conventional Amphotericin B) used to treat invasive fungal infection in AIDS patient.(Moen et al., 2009). Liposomal Amphotericin B composed of accumulation of liposomes at the site of fungal infection and causes death to cells.(Coukell\& Brogden, 1998). Anidulafungin is an antifungal agent which belongs to the class of Echinocandins are used to inhibit the fungal cell wall synthesis by the enzyme beta - (1,3) D glucan synthase.(Murdoch \& Plosker, 2004). Terbinafine, itraconazole, fluconazole are given as a ointments are used to cure skin infections in nails and skin.(Gupta et al., 2005).

\section{Combination of antifungal agents}

Antifungal drug with the combination of anti-virulence drugs is use to inhibit the growth of Candida albicans by destroying the synthesis of cell membrane of fungi "ergosterol". Echinocandins are mostly used to treat Candidiasis. (Cui et al., 2015). Graphene oxide is biocompatibility agent and graphene are composed of hydroxyl, carbonyl, carboxylic compound to construct new composite made up to nano medicine. Those graphene compound with the combination of fluconazole for the treatment of Candidiasis. (Asadi Shahi et al., 2019). Statins are cholesterol lowering agent which has antifungal properties to treat the fungal infections in human. Lovastatin and Simvastatin with the combination of azole, Amphotericin B inhibit the growth of Aspergillus niger and Candida albicans. (Asadi Shahi et al., 2019).

N-butylphthalide is extracted from the seed of Apium graveolens (flowering plants belongs to Apiaceae family) had antifungal property. N-butylphthalide with the combination of fluconazole is used treat fungal infections by Candida albicans. (Ying Gong et al., 2019). Nystatin combined with chlorhexidine are used as oral medicine of single formulation which are to treat fungal infections. It was analysed by minimum inhibitory growth in Candida suspensions. The mixture of Nystatin and Chlorhexidine tested by using High performance lipid Chromatography. As a result, the combination of Nystatin and Chlorhexidine has antifungal activity and used to treat Candidiasis in human. (Scheibler et al., 2018). Combination of five antifungal agents like Fluconazole, Voriconazole, Caspofungin, Nystatin, Amphotericin B with cyclosporine A were tested against Candida albicans by using XTT metabolic assay. (Shinde et al., 2012). The combination of Ginkgolide B and fluconazole were tested by minimum inhibitory growth on Candida biofilms. As a result, the combination of those drugs inhibited the synthesis of fungal hyphae. (Li et al., 2020). Micafungin, anidulafungin, caspofungin are belongs to the class of echinocandin used to target the beta 1,3-glucan synthesis of cell 
wall for the treatment of invasive Aspergillosis. (Aruanno et al., 2019). Combination of geldanamycin with fluconazole were tested on Candida albicans strain by using antibiotic sensitivity test. As a result, geldanamycin with fluconazole inhibited the growth of Candida albicans. (Lu et al., 2017). An organic chemical compound "Pneumocandin $\mathrm{B}_{0}$ " and hydroxy echinocandin which can be synthesised by the fungus Glarealozoyensisis the strong antifungal agent and inhibit the synthesis of beta -1,3 D glucan cell wall of Candida albicans. It is mainly used to treat fungal infections in nails and skin during organ transplant and in AIDS patient. (Martín Mazuelos \& Rodríguez-Tudela, 2008).

Natural compounds like ajoene (extracts of garlic), allicin (extracts of chopped garlic), curcumin (extracts of turmeric), gingerol (phenolic compound found in ginger), nimbin (extracts of neem oil), nimbolide (leaf extract of Azadirachta indica), nimonol (leaf extract of Azadirachta indica), 6-shogaol (pungent constituents of ginger). These natural compounds have been docked against sterol-14-demthylase, cytochrome $\mathrm{P}_{450}$, squalene monooxygenase, lanosterol monooxygenase.(Srivastava, Srivastava - 2020).

\section{Comparison and effectiveness of antifungal agents}

In vitro activity of LY303366 (LY) against Candida albicans strains and compared with fluconazole (FLU), flucytosine (5FC) and amphotericin B (AMB) by using microtitre modification method and minimal fungal concentrations can be detected. As a result, new echinocandin of LY303366 showed good antifungal activity when compared to fluconazole, flucytosine and amphotericin B (Moore et al., 2000). MK-0991 (in the past L-743,872) is a water solvent semisynthetic echinocandin that has intense antifungal activity compared with itraconazole, fluconazole, amphotericin B, 5flucytosine evaluated against candida strains in the blood stream. Minimum inhibitory concentration of antifungal agents analysed by using RPMI 1640 test medium. As a result, semisynthetic echinocandin highly inhibits the growth of Candida albicans when compared to fluconazole, amphotericin B, 5-flucytosine (Marco et al., 1998). The essential oils which are extracted from plant-like cinnamon, anise, clove, citronella, peppermint, pepper, camphor is tested against agar diffusion assay containing Aspergillus niger strains. As a result, cinnamon, clove showed highest inhibition than other essential oils (Hu et al., 2019).Natural products like garlic (Allium sativum Linn), ginger (Zingiber officinale Roscoe), and lime (Citrus aurantifolia Linn) are usually having medicinal property to cure any infections in human. These natural products are extracted by using an ethanol extract medium and tested on PDA control. As, a result, all the natural compounds inhibited the growth of Aspergillus niger. When compared all these, essential oil from ginger is more effective (Nii et al., 2010).

The antifungal activities of ketoconazole were investigated both In vitro and in vivo. The antifungal efficiency of ketoconazole In vitro was studied in Sabouraud's broth including Eagle's minimal essential medium against fungal strains of Candida albicans and other Candida spp. Ketoconazole is highly active In vitro and possesses broad spectrum activity. Ketoconazole's activity is increased, because Eagle's minimal essential medium is enriched in serum. Ketoconazole is very potent in the topical treatment skin candidiasis, and in vaginal candidiasis of laboratory animals. Ketoconazole is superior to griseofulvin in the oral treatment of skin dermatophytosis. No side effects are observed.(Van Cutsem, 1983).

Boric acid was compared with nystatin, terconazole, flucytosine, itraconazole, clotrimazole, ketoconazole, fluconazole, buconazole, and miconazole; as monotherapy, boric acid was analysed against Candida albicans. As a result, boric acid is most effective than other antifungal drugs. Boric acid is inhibited the growth of Candida albicans. The adverse effects of boric acid are vaginal burning sensation, water discharge during treatment, and vaginal erythema were identified (Iavazzo et al., 2011).Fluconazole is used to treat candidiasis infections like oral candidiasis. Intravenous amphotericin $\mathrm{B}$ is used to treat vaginal candidiasis and oral candidiasis in AIDS patients. So, these drugs are compared to nystatin and clotrimazole. Clotrimazole is most effective when compared to other drugs were analysed (Albougy \& Naidoo, 2002).Voriconazole, natamycin, itraconazole, clotrimazole, econazole, ketoconazole is compared and tested against Aspergillus spp which are isolated by keratits or corneal ulcer patients. These antifungal compounds are used to compare by using minimum inhibitory concentration. As a result, ketoconazole is more effective against Aspergillus infections in humans. Ketoconazole is more effective and inhibit the growth of fungi in humans.

\section{Side effects of antifungal agents}

\subsection{Side effects of topical antifungal agents}

- Skin rashes

- Irritation

- Itching 
- Burning sensation

- Redness.

\subsection{Side effects of oral antifungal agents like capsules causes}

- Indigestion

- Abdominal pain

- Headache

- Rashes

- Feeling sick

- Overdosage of oral antifungal agents causes allergic reactions like swelling of neck, face, tongue and also causes difficulty in breathing and also causes peeling of skin or formation of blisters in skin.

- It also causes liver damage and the symptoms are,

- Jaundice (yellow fever)

- Loss of appetite

- 10)Vomiting(Thompson Iii et al., 2009)

\section{Conclusion}

Candidiasis is the fungal infection caused by Candida albicans. It causes different type of candidiasis infections in the blood, heart, eyes, brain, bones also other parts of our body. It can be cured by using natural compounds or synthetic compounds or semisynthetic compounds. Apergillosis is the fungal infections in human caused by Aspergillus niger. Aspergillosis infections can be cured by using synthetic compounds like intravenous injections, oral medicines of capsules, tropical medicines like creams and some other natural products from plants.Natural products like saponins, flavonoids, alkaloids, xanthones, lectins and polypeptides, quinones, terpenoids, coumarins, essential oils and other compounds are the secondary metabolites extracted from the plants. Those plants are effective against fungal infections. Synthetic drugs like Azole group of drugs composed of imidazoles which includes miconazole, clotrimazole, ketoconazole, and triazoles include itraconazole, Posaconazole, isavuconazole are used to inhibit the ergosterol synthesis of cell membrane of fungiand as a result ergosterol can be inhibited. Combination of drugs like natural products and synthetic produced has been used. Natural compounds like ajoene (extracts of garlic), allicin (extracts of chopped garlic), curcumin (extracts of turmeric), gingerol (phenolic compound found in ginger), nimbin (extracts of neem oil), nimbolide (leaf extract of Azadirachta indica), nimonol (leaf extract of Azadirachta indica), 6-shogaol (pungent constituents of ginger). These natural compounds have been docked against sterol-14-demthylase, cytochrome P450, squalene monooxygenase, lanosterol monooxygenase. Comparison and effectiveness of drugs depends upon the infections they are getting affected. Amphotericin B and azole group of drugs are commonly more effective than other group of drugs, but ketoconazole is widely used. Adverse effects of antifungal agents include, skin rashes, irritation, itching, nausea and vomiting, fever, abdominal pain, muscle and joint pain, etc.

\section{Compliance with ethical standards}

\section{Acknowledgments}

I thank my guide Dr. Priya Iyer, Head of the department of PG \& Research department of Biotechnology, for her support throughout the project. I owe thanks to Dr. Anitha RJ Singh, Dr. A. Pavithra and Dr. Anchana Devi for their support. I thank the Management of Women's Christian College and Dr. Lilian I. Jasper, Principal of Women's Christian College for providing this opportunity.

\section{Disclosure of conflict of interest}

The authors declare that there is no conflict of interest exit.

\section{References}

[1] Agarwal R, Sehgal IS, Dhooria S, Aggarwal AN. Developments in the diagnosis and treatment of allergic bronchopulmonary aspergillosis. In Expert Review of Respiratory Medicine. 2016; 10(12): 1317-1334.

[2] Albougy HA, Naidoo S. A systematic review of the management of oral candidiasis associated with HIV/AIDS. In SADJ: journal of the South African Dental Association = tydskrif van die Suid-Afrikaanse Tandheelkundige Vereniging. 2002; 57(11): 457-466. 
[3] Antifungal flavonoid from Alpinia officinarum Hance - PubMed. (n.d.).

[4] Arif T, Bhosale JD, Kumar N, Mandal TK, Bendre RS, Lavekar GS, Dabur R. Natural products - Antifungal agents derived from plants. In Journal of Asian Natural Products Research. 2009; 11(7): 621-638.

[5] Aruanno M, Glampedakis E, Lamoth F. Echinocandins for the treatment of invasive aspergillosis: From laboratory to bedside. In Antimicrobial Agents and Chemotherapy. American Society for Microbiology. 2019; 63(8).

[6] Asadi Shahi S, Roudbar Mohammadi S, Roudbary M, Delavari H. A new formulation of graphene oxide/fluconazole compound as a promising agent against Candida albicans. Progress in Biomaterials. 2019; 8(1): 43-50.

[7] Bakshi SS. Erythematous Candidiasis. Journal of Allergy and Clinical Immunology: In Practice. 2018 ; $6(2): 646$.

[8] Belhouchet Z, Sautour M, Miyamoto T, Lacaille-Dubois MA. Steroidal saponins from the roots of Smilax aspera subsp. mauritanica. Chemical and Pharmaceutical Bulletin. 2008; 56(9): 1324-1327.

[9] Bersani I, Piersigilli F, Goffredo BM, Santisi A, Cairoli S, Ronchetti MP, Auriti C. Antifungal drugs for invasive candida infections (ICI) in neonates: Future perspectives. In Frontiers in Pediatrics. Frontiers Media S.A. SEP 2019; 7: 375.

[10] Bodey GP. Azole Antifungal Agents. Clinical Infectious Diseases. 1992; 14(1): S161-S169.

[11] Boukhatem MN, Ferhat MA, Kameli A, Saidi F, Kebir HT. Lemon grass (cymbopogon citratus) essential oil as a potent anti-inflammatory and antifungal drugs. Libyan Journal of Medicine. 2014; 9.

[12] Chakrabarti A, Kaur H. Allergic Aspergillus rhinosinusitis. In Journal of Fungi MDPI AG. 2016; 2(4).

[13] Chu KT, Liu KH, Ng TB. Cicerarin, a novel antifungal peptide from the green chickpea. Peptides. 2003; 24(5): 659663.

[14] Clerihew L, McGuire W. Antifungal therapy for newborn infants with invasive fungal infection. Cochrane Database of Systematic Reviews. 2012; 6: CD003953-CD003953.

[15] Coukell AJ, Brogden RN. Liposomal amphotericin B: Therapeutic use in the management of fungal infections and visceral leishmaniasis. Drugs. 1998; 55(4): 585-612.

[16] Cui J, Ren B, Tong Y, Dai H, Zhang L. Synergistic combinations of antifungals and anti-virulence agents to fight against Candida albicans. Virulence. 2015; 6(4): 362-371.

[17] Denning DW, Marr KA, Lau WM, Facklam DP, Ratanatharathorn V, Becker C, Ullmann AJ, Seibel NL, Flynn PM, van Burik JAH, Buell DN, Patterson TF. Micafungin (FK463), alone or in combination with other systemic antifungal agents, for the treatment of acute invasive aspergillosis. Journal of Infection. 2006; 53(5): 337-349.

[18] Dharmaratne HRW, Piyasena KGNP, Tennakoon SB. A geranylated biphenyl derivative from Garcinia mangostana. Natural Product Research. 2005; 19(3): 239-243.

[19] Enoch DA, Murphy ME, Micallef C, Yang H, Brown NM, Aliyu SH. Micafungin use in a UK tertiary referral hospital. Journal of Global Antimicrobial Resistance. 2018; 15: 82-87.

[20] Greenberger PA, Bush RK, Demain JG, Luong A, Slavin RG, Knutsen AP. Allergic Bronchopulmonary Aspergillosis. Journal of Allergy and Clinical Immunology: In Practice. 2014; 2(6): 703-708.

[21] Grover N. Echinocandins: A ray of hope in antifungal drug therapy. In Indian Journal of Pharmacology. 2010; 42(1): 9-11. Wolters Kluwer -- Medknow Publications.

[22] Gupta AK, Ryder JE, Chow M, Cooper EA. Dermatophytosis: the management of fungal infections. In Skinmed. 2005; 4(5): 305-310. Skinmed.

[23] Haidara M, Bourdy G, De Tommasi N, Braca A, Traore K, Giani S, Sanogo R. Medicinal Plants Used in Mali for the Treatment of Malaria and Liver Diseases. Natural Product Communications. 2016; 11(3): $1934578 X 1601100$.

[24] Hani U, Shivakumar H, Vaghela R, M Osmani R, Shrivastava A. Candidiasis: A Fungal Infection- Current Challenges and Progress in Prevention and Treatment. Infectious Disorders - Drug Targets. 2015; 15(1): 42-52.

[25] Hay AE, Guilet D, Morel C, Larcher G, Macherel D, Le Ray AM, Litaudon M, Richomme P. Antifungal Chromans Inhibiting the Mitochondrial Respiratory Chain of Pea Seeds and New Xanthones from Calophyllum caledonicum. Planta Medica. 2003; 69(12): 1130-1135.

[26] HAYTA Ş, YAZGIN A, BAĞCI E. Composition of the essential oils of two Teucrium species from Turkey. Bitlis Eren University Journal of Science and Technology. 2017; 7(2): 140-144. 
[27] Hellstein JW, Marek CL. Candidiasis: Red and White Manifestations in the Oral Cavity. In Head and Neck Pathology. 2019; 13(1): 25-32. Humana Press Inc.

[28] Hu F, Tu XF, Thakur K, Hu F, Li XL, Zhang YS, Zhang JG, Wei ZJ. Comparison of antifungal activity of essential oils from different plants against three fungi. Food and Chemical Toxicology. 2019; 134.

[29] Iavazzo C, Gkegkes ID, Zarkada IM, Falagas ME. Boric acid for recurrent vulvovaginal candidiasis: The clinical evidence. In Journal of Women's Health. 2011; 20(8): 1245-1255. J Womens Health (Larchmt).

[30] Janeczko M. Emodin reduces the activity of $(1,3)-\beta$-D-glucan synthase from candida albicans and does not interact with caspofungin. Polish Journal of Microbiology. 2018; 67(4): 463-470.

[31] Katzenstein ALA, Sale SR, Greenberger PA. Allergic Aspergillus sinusitis: a newly recognized form of sinusitis. The Journal of Allergy and Clinical Immunology. 1983; 72(1): 89-93.

[32] Kauffman CA. Diagnosis and management of fungal urinary tract infection. In Infectious Disease Clinics of North America. 2014; 28(1): 61-74. Infect Dis Clin North Am.

[33] Kobashi Y, Fukuda M, Yoshida K, Miyashita N, Niki Y, Oka M. Chronic necrotizing pulmonary aspergillosis as a complication of pulmonary Mycobacterium avium complex disease. Respirology. 2006; 11(6): 809-813.

[34] Kontoyiannis DP, Reddy BT, Torres HA, Luna M, Lewis RE, Tarrand J, Bodey GP, Raad II. Pulmonary candidiasis in patients with cancer: An autopsy study. Clinical Infectious Diseases. 2002; 34(3): 400-403.

[35] Korting HC, Schöllmann C. The significance of itraconazole for treatment of fungal infections of skin, nails and mucous membranes. In JDDG - Journal of the German Society of Dermatology. 2009; 7(1): 11-19. Blackwell Publishing Ltd.

[36] Lam SK, Ng TB. Lectins: Production and practical applications. In Applied Microbiology and Biotechnology. 2011; 89(1): 45-55. Nature Publishing Group.

[37] Lavault M, Landreau A, Larcher G, Bouchara JP, Pagniez F, Le Pape P, Richomme P. Antileishmanial and antifungal activities of xanthanolides isolated from Xanthium macrocarpum. Fitoterapia. 2005; 76(3-4): 363-366.

[38] Lelarge P, Mariot J. Candidoses systémiques. In Annales francaises d'anesthesie et de reanimation. 1992; 11(5): 558-575. Ann Fr Anesth Reanim.

[39] Leli C, Mencacci A, Meucci M, Bietolini C, Vitali M, Farinelli S, D’Alò F, Bombaci JC, Perito S, Bistoni F. Association of pregnancy and Candida vaginal colonization in women with or without symptoms of vulvovaginitis. Minerva Ginecologica. 2013; 65(3): 303-309.

[40] Li Y, Yang J, Li X, Su S, Chen X, Sun S, Li Y. The effect of Ginkgolide B combined with fluconazole against drugresistant Candida albicans based on common resistance mechanisms. International Journal of Antimicrobial Agents. 2020; 56(2).

[41] Lu M, Li T, Wan J, Li X, Yuan L, Sun S. Antifungal effects of phytocompounds on Candida species alone and in combination with fluconazole. In International Journal of Antimicrobial Agents. 2017; 49(2): 125-136. Elsevier B.V.

[42] Marco F, Pfaller MA, Messer SA, Jones RN. Activity of MK-0991 (1-743,872), a new echinocandin, compared with those of LY303366 and four other antifungal agents tested against blood stream isolates of Candida spp. Diagnostic Microbiology and Infectious Disease. 1998; 32(1): 33-37.

[43] Martín Mazuelos E, Rodríguez-Tudela JL. In vitro activity of anidulafungin. Comparison with the activity of other echinocandins. Enfermedades Infecciosas y Microbiologia Clinica. 2008; 26(14): 7-13.

[44] Martins N, Ferreira ICFR, Barros L, Silva S, Henriques M. Candidiasis: Predisposing Factors, Prevention, Diagnosis and Alternative Treatment. In Mycopathologia. 2014; 177: 5-6, 223-240. Kluwer Academic Publishers.

[45] McCarty TP, Pappas PG. Invasive Candidiasis. In Infectious Disease Clinics of North America. 2016; 30(1).

[46] McNaught AD, Wilkinson A, IUPAC. flavonoids (isoflavonoids and neoflavonoids). In IUPAC Compendium of Chemical Terminology (2nd ed.). IUPAC. 2008.

[47] Meis JFGM, Verweij PE. Current management of fungal infections. Drugs. 2001; 61(1): 13-25.

[48] Meragelman TL, Tucker KD, McCloud TG, Cardellina JH, Shoemaker RH. Antifungal flavonoids from Hildegardia barteri. Journal of Natural Products. 2005; 68(12): 1790-1792. 
[49] Moen MD, Lyseng-Williamson KA, Scott LJ. Liposomal amphotericin B: A review of its use as empirical therapy in febrile neutropenia and in the treatment of invasive fungal infections. In Drugs. 2009; 69(3): 361-392. Drugs.

[50] Mohamed AA, Lu XL, Mounmin FA. Diagnosis and Treatment of Esophageal Candidiasis: Current Updates. In Canadian Journal of Gastroenterology and Hepatology. 2019. Hindawi Limited.

[51] Moore CB, Oakley KL, Denning DW. In vitro activity of a new echinocandin, LY303366, and comparison with fluconazole, flucytosine and amphotericin B against Candida species. Clinical Microbiology and Infection. 2000; 7(1): 11-16.

[52] Murdoch D, Plosker GL. Anidulafungin. In Drugs. 2004; 64(19): 2249-2258. Drugs.

[53] Nii D, Tagoe A, Essien-Baidoo S, Kangah V. A Comparison Of The Antimicrobial (Antifungal) Properties Of Garlic, Ginger And Lime On Aspergillus Flavus, Aspergillus Niger And Cladosporium Herbarum Using Organic And Water Base Pharmacognostic and physico-chemical investigation of Palisota hirsuta (K. Schum) (Commelinaceae) View project Epidemiology and Infectious Diseases View project. In Article in Internet Journal of Tropical Medicine. 2010; 24.

[54] Nittayananta W, Limsuwan S, Srichana T, Sae-Wong C, Amnuaikit T. Oral spray containing plant-derived compounds is effective against common oral pathogens. Archives of Oral Biology. 2018; 90: 80-85.

[55] Novak B, Hudlicky T, Reed J, Mulzer J, Trauner D. Morphine Synthesis and Biosynthesis-An Update. Current Organic Chemistry. 2005; 4(3): 343-362.

[56] Panagakos FS, Migliorati CA. Diagnosis and Management of Oral Lesions and Conditions: A Resource Handbook for the Clinician. In Diagnosis and Management of Oral Lesions and Conditions: A Resource Handbook for the Clinician. InTech. 2014.

[57] Papon N, Courdavault V, Clastre M, Bennett RJ. Emerging and Emerged Pathogenic Candida Species: Beyond the Candida albicans Paradigm. PLoS Pathogens. 2013; 9(9): e1003550.

[58] Patai S, Rappoport Z. The Chemistry of the Quinonoid Compounds. In The Chemistry of the Quinonoid Compounds. 2010; 2. wiley.

[59] Pettit, N. N., \& Carver, P. L. Isavuconazole: A New Option for the Management of Invasive Fungal Infections. Annals of Pharmacotherapy. 2015; 49(7): 825-842.

[60] Peyton LR, Gallagher S, Hashemzadeh M. Triazole antifungals: A review. In Drugs of Today. 2015; 51(12): 705718. Prous Science.

[61] Pfaller MA, Messer S, Jones RN. Activity of a new triazole, Sch 56592, compared with those of four other antifungal agents tested against clinical isolates of Candida spp. and Saccharomyces cerevisiae. Antimicrobial Agents and Chemotherapy. 1997; 41(2): 233-235.

[62] Rachwalski EJ, Wieczorkiewicz JT, Scheetz MH. Posaconazole: An oral triazole with an extended spectrum of activity. In Annals of Pharmacotherapy. 2008; 42(10): 1429-1438. Ann Pharmacother.

[63] Rawashdeh MA, Ayesh JAM, Darwazeh AMG. Oral candidal colonization in cleft patients as a function of age, gender, surgery, type of cleft, and oral health. Journal of Oral and Maxillofacial Surgery. 2011; 69(4): 1207-1213.

[64] Rhew ZI, Han Y. Synergic effect of combination of glycyrol and fluconazole against experimental cutaneous candidiasis due to Candida albicans. Archives of Pharmacal Research. 2016; 39(10): 1482-1489.

[65] Saag MS, Dismukes WE. Azole antifungal agents: emphasis on new triazoles. Antimicrobial Agents and Chemotherapy. 1988; 32(1).

[66] Sambatakou H, Dupont B, Lode H, Denning DW. Voriconazole Treatment for Subacute Invasive and Chronic Pulmonary Aspergillosis. American Journal of Medicine. 2006; 119(6): 527.e17-527.e24.

[67] Sardari S, Mori Y, Horita K, Micetich RG, Nishibe S, Daneshtalab M. Synthesis and antifungal activity of coumarins and angular furanocoumarins. Bioorganic and Medicinal Chemistry. 1999; 7(9): 1933-1940.

[68] Scheibler E, da Silva RM, Leite CE, Campos MM, Figueiredo MA, Salum FG, Cherubini K. Stability and efficacy of combined nystatin and chlorhexidine against suspensions and biofilms of Candida albicans. Archives of Oral Biology. 2018; 89: 70-76.

[69] Scott LJ, Simpson D. Voriconazole: A review of its use in the management of invasive fungal infections. In Drugs. 2007; 67(2): 269-298. Drugs. 
[70] Shinde RB, Chauhan NM, Raut JS, Karuppayil SM. Sensitization of Candida albicans biofilms to various antifungal drugs by cyclosporine A. Annals of Clinical Microbiology and Antimicrobials. 2012; 11(1): 27.

[71] Smith WJ, Drew RH, Perfect JR. Posaconazole's impact on prophylaxis and treatment of invasive fungal infections: An update. Expert Review of Anti-Infective Therapy. 2009; 7(2): 165-181.

[72] Spampinato C, Leonardi D. Candida infections, causes, targets, and resistance mechanisms: Traditional and alternative antifungal agents. In BioMed Research International. 2013.

[73] Srivastava A, Srivastava A, \& -, N. In-silico study of some natural compounds used as antifungal agents against Candida albicans. European Journal of Biological Research. 2020; 10(3): 188-197.

[74] Thompson Iii GR, Cadena J, Patterson TF. Fungal infection Invasive mycoses Triazoles Echinocandins Amphotericin Flucytosine Clin Chest Med. Clinics in Chest Medicine. 2009; 30: 203-215.

[75] Van Cutsem J. The antifungal activity of ketoconazole. The American Journal of Medicine. 1983; 74(1-2): 9-15.

[76] Vila T, Sultan AS, Montelongo-Jauregui D, Jabra-Rizk MA. Oral candidiasis: A disease of opportunity. In Journal of Fungi. 2020; 6(1).

[77] Willems L, Van Der Geest R, De Beule K. Itraconazole oral solution and intravenous formulations: A review of pharmacokinetics and pharmacodynamics. In Journal of Clinical Pharmacy and Therapeutics. 2001; 26(3): 159169). J Clin Pharm Ther.

[78] Ying Gong, Liu W, Huang X, Hao L, Li Y, Sun S. Antifungal activity and potential mechanism of n-butylphthalide alone and in combination with fluconazole against Candida albicans. Frontiers in Microbiology, 10(JUL). 2019.

[79] Zmeili OS, Soubani AO. Pulmonary aspergillosis: A clinical update. In QJM. 2007; 100(6): 317-334. 